\title{
A prospective randomized study: role of enalapril on oxidative stress in newly diagnosed type 2 diabetes mellitus and its correlation with glycaemic status
}

\author{
Sathiya Vinotha A. T. ${ }^{*}$, Ranjani R. ${ }^{2}$
}

${ }^{1}$ Department of Pharmacology, Karpagam Faculty of Medical Sciences and Research, Coimbatore, Tamil Nadu, India ${ }^{2}$ Department of Pharmacology, Chengalpattu Medical College, Chengalpattu, Tamil Nadu, India

Received: 20 January 2017 Accepted: 27 February 2017

\section{*Correspondence to:}

Dr. Sathiya Vinotha A.T., Email: drs.vinotha@gmail.com

Copyright: (C) the author(s), publisher and licensee Medip Academy. This is an openaccess article distributed under the terms of the Creative Commons Attribution NonCommercial License, which permits unrestricted noncommercial use, distribution, and reproduction in any medium, provided the original work is properly cited.

\begin{abstract}
Background: Oxidative stress is demonstrated the pathological conditions such as Atherosclerosis and diabetes mellitus. It has been suggested to be involved in the pathogenesis of both macro and microvascular complications. This study was designed to evaluate the effect of Enalapril on oxidative stress markers in type 2 diabetic patients.

Methods: A Prospective Randomized controlled study was conducted in 75 participants in which 25 healthy volunteers and 50 newly diagnosed DM2 patients who were divided into three groups of 25 in each. In Group II DM2 patients were treated with Metformin alone, in group III DM2 patients were treated with Enalapril and Metformin and 25 healthy volunteers were included in Group I (controls). Oxidative stress parameters, FBS, HbA1c were measured at the time of diagnosis and at 4,8,12 weeks.

Results: There was a significant decrease in free radical production $(p<0.05)$ and significant increase in antioxidant enzymes $(p<0.001)$ in both the patient Groups and positive correlation between oxidative stress markers and FBS and HbA1c $(\mathrm{p}<0.01)$.

Conclusions: Oxidative stress measured as LPO is strictly influenced by glycaemic control. Enalapril and Metformin combination in diabetic patients has more significant effect on decreasing the oxidative stress than Metformin alone.
\end{abstract}

Keywords: ACE inhibitors, Biguanides, Glycated haemoglobin, Reactive oxygen species

\section{INTRODUCTION}

Diabetes mellitus (DM) is one of the most common noncommunicable disease worldwide, with over $80 \%$ of its carriers living in low- and middle-income countries. The incidence of type $2 \mathrm{DM}$ is increasing in part to a western style diet, increasing obesity, sedentary life style. More than $90 \%$ of DM are of Type 2 which commonly affects persons over 40 years old and leads to various macrovascular and microvascular complications. ${ }^{1}$

The pathogenesis of diabetes mellitus is due to insulin resistance and impaired insulin secretion which leads to hyperglycemia and compensatory hyperinsulinemia.
The present investigations are focused on two mechanisms for insulin resistance in Type $2 \mathrm{DM}$, one is accumulation of lipid in liver and muscle and secondly due to obesity induced inflammation. ${ }^{2}$ Both these mechanisms lead to generation of more free radicals, causes imbalance between generation and elimination of Reactive Oxygen Species (ROS) results in oxidative stress.

Defective mitochondrial metabolism, glucose autooxidation, NADPH oxidation and synthesis of advanced glycation products are several sources of ROS in diabetes. $^{3}$ ROS may also act as transduction signal for angiotensin-II in different cell types, like smooth muscle 
cells, endothelial cells and human ventricular myocytes. The type 2 diabetes mellitus usually manifests with elevated fasting blood sugar secondary to insufficient insulin action. ${ }^{3,4}$ The United Kingdom Prospective Diabetes Study (UKPDS) demonstrated a progressive decline of endogenous insulin release and demonstrated $\beta$-cell function of less than $60 \%$ at baseline for patients with type 2 Diabetes mellitus. ${ }^{5}$ Glycated haemoglobin (HbA1c) is a form of haemoglobin used to measure the average plasma glucose concentration over prolonged period of time. It is formed in a non-enzymatic pathway by haemoglobin reaction to high plasma levels of glucose. Glycated haemoglobin has been associated with cardiovascular disease, nephropathy, and retinopathy in diabetes mellitus. Glycated haemoglobin is recommended for checking blood sugar control in people who might be pre-diabetic and monitoring blood sugar control in patients with more elevated levels. ${ }^{6,7}$

The primary goal of type $2 \mathrm{DM}$ treatment is to achieve and maintain good glycemic control, and to reduce the mortality and risk of microvascular and macrovascular complications . The current management of type $2 \mathrm{DM}$ is a combination of lifestyle intervention and Metformin as initial therapy for type $2 \mathrm{DM}$, followed by other Oral Hypoglycemic Agents and Insulin. ${ }^{8}$ Besides Biguanides (Metformin), other antidiabetic agents include Sulfonylureas, Glitinides, Thiazolidinediones or Glitazones, $\alpha$-glucosidase inhibitors (Acarbose), GLP1analogues, Dipeptidyl peptidase 4 inhibitors, Amylin agonists (Pramlintide) and Sodium glucose co transport-2 inhibitors (Dapaglifozin). Therapeutic profile of Metformin has been evaluated for more than five decades and till now it is the most commonly used drug in type 2 DM. Experimental and clinical studies have shed new light on the multiple beneficial effects of this drug, not only in the treatment of diabetes. ${ }^{8,9}$

A number of reports have shown that antioxidants can attenuate the complications of diabetes in patients and in experimental model. ${ }^{10}$ ACE inhibitors have been shown to attenuate the progression of cardiac and renal impairments related to diabetes and to reduce the risk of death in diabetic patients. ${ }^{11}$ The beneficial actions of ACE inhibitors seem to be independent of their effect on blood pressure, but it is not fully understood how they provide such protection.

Some studies have focused on their in vitro antioxidant properties, and others have shown that chronic treatment with ACE inhibitors can increase the endogenous antioxidant defences in healthy animals and in patients with end-stage renal disease under hemodialysis therapy. ${ }^{12,13}$ Other studies have pointed to the possible protective antioxidant action of ACE inhibitors in the blood and kidneys of Streptozotocin (STZ)-induced diabetic rats. ${ }^{14}$ It is well known that ACE inhibitors does not decrease BP in normotensives. In the present study, we investigated the effect of Metformin and Enalapril on oxidative damage in newly diagnosed human DM2 patients and relationship between glycaemic control, and oxidative stress.

\section{METHODS}

The study was conducted after having obtained Ethical clearance from Human Ethical Committee at Rajiv Gandhi Government General Hospital, Madras Medical College, Chennai. Informed consent in the prescribed form was obtained from all patients included in the study after explanation of the probable cons and pros in local language.

\section{Study design and participants}

A Randomized controlled study was conducted in 75 participants in which 25 healthy volunteers and 50 newly diagnosed DM2 patients who were divided into three groups of 25 in each. In Group II DM2, patients were treated with Metformin alone, in group III DM2 patients were treated with Enalapril and Metformin and 25 healthy volunteers were included in Group I (controls).

\section{Inclusion criteria}

Patient age >45 years, Both male and female, Newly diagnosed diabetic patients with FBS (fasting blood sugar) $\geq 126 \mathrm{mg} / \mathrm{dl}, \mathrm{HbA1}_{\mathrm{c}}$ (glycosylated haemoglobin) $>6.5 \%$.

\section{Exclusion criteria}

Patients those who are not willing to give consent, Pregnant women and children, Patients with diabetes and hypertension on treatment with other drugs, Patients with H/O Stroke, Asthma, Patients with hypertension, Patients with renal, liver failure and Myocardial infarction.

\section{Blood collection and laboratory methods}

Blood sample was collected under aseptic precautions at the time of diagnosis and at 4 weeks interval for a period of 12 weeks. The biochemical profile was evaluated by using standard laboratory methods. Samples were kept cool until the completion of blood collection and then centrifuged at $4000 \mathrm{rpm}$, at $4^{\circ} \mathrm{C}$ for 5 minutes.

The serum was separated and analyzed for Catalase (method of Sinha), reduced glutathione (method of Ellman), GST (method of Habig et al), Superoxide dismutase (method of Marklund and Marklund) and free radical production was measured by lipid peroxidation (method of Ohkawa et al). Protein content was measured by method of Lowry et al. All these parameters were determined by using UV (ultraviolet) spectrophotometer. Bio-Rad IN2IT (1) Analyser kit produced by Bio-Rad Laboratories Inc.UK was used for estimation of glycated haemoglobin, and Enzymatic- colorimetric estimation of plasma fasting sugar was done using the glucose oxidaseperioxidase method. ${ }^{15,16}$ 


\section{Statistical analysis}

The results were expressed as mean \pm standard deviation (mean \pm SD). Statistical significance of difference between control, patient groups was evaluated using one way ANOVA. All calculations were performed using the SPSS version 20.0 for windows.

\section{RESULTS}

When compared from 0 to 12 weeks there is significant increase in all antioxidants Catalase, GSH, GST in
Groups II and III $(p<0.001)$ and SOD $(p<0.05)$ but less than Group I (healthy volunteers). LPO shows significant increase in both the groups (II and III) when compared with controls (Group I) at 0 week and showed significant decrease from 0 to 12 weeks $(\mathrm{p}<0.05)$. Group II shows more significant improvement in all antioxidant levels and decrease in LPO levels than Group III $(\mathrm{p}<0.01)$.

The LPO level and antioxidants correlated positively with fasting blood sugar (FBS) and glycated haemoglobin $\left(\mathrm{HbA}_{1} \mathrm{c}\right)$ between the 0 week and 12 week $(\mathrm{p}<0.01)$. All the results were clearly shown in Table 1.

Table 1: Effect of enalapril and metformin on antioxidant levels and glycaemic status in diabetic patients.

\begin{tabular}{|c|c|c|c|c|c|}
\hline Enzymes & Groups & 0 Week & 4 Week & 8 Week & 12 Week \\
\hline \multirow{3}{*}{ Catalase } & GroupI & $83.00 \pm 9.74$ & $82.54 \pm 9.01$ & $82.89 \pm 8.83$ & $82.94 \pm 8.83$ \\
\hline & Group II & $58.03 \pm 7.56$ & $58.14 \pm 8.63 *$ & $58.29 \pm 8.63 *$ & $58.65 \pm 8.44 * * *$ \\
\hline & GroupIII & $58.11 \pm 6.33$ & $58.35 \pm 6.29 *$ & $58.51 \pm 6.29 *$ & $58.68 \pm 6.19 * * *$ \\
\hline \multirow{3}{*}{ Reduced Glutathione } & GroupI & $13.20 \pm 1.05$ & $12.31 \pm 1.13$ & $13.18 \pm 1.04$ & $13.19 \pm 1.04$ \\
\hline & Group II & $10.60 \pm 1.88$ & $11.09 \pm 1.80 * * *$ & $11.69 \pm 1.83 * * *$ & $11.71 \pm 1.83 * * *$ \\
\hline & GroupIII & $9.73 \pm 1.08$ & $9.86 \pm 1.15^{*}$ & $10.00 \pm 1.18 * * *$ & $10.03 \pm 1.19 * * *$ \\
\hline \multirow{3}{*}{ Glutathione transferase } & GroupI & $18.94 \pm 2.69$ & $18.98 \pm 2.20$ & $19.11 \pm 2.68$ & $19.12 \pm 2.69$ \\
\hline & Group II & $11.77 \pm 1.61$ & $12.03 \pm 1.75^{* *}$ & $12.65 \pm 1.94 * *$ & $12.68 \pm 1.94 * * *$ \\
\hline & GroupIII & $12.76 \pm 1.17$ & $13.88 \pm 1.06^{* * *}$ & $14.18 \pm 1.27 * * *$ & $16.21 \pm 1.26^{* * *}$ \\
\hline \multirow{3}{*}{ SOD } & GroupI & $2.93 \pm 0.36$ & $2.94 \pm 0.24$ & $2.90 \pm 0.32$ & $2.92 \pm 0.31$ \\
\hline & Group II & $2.37 \pm 0.52$ & $2.52 \pm 0.50 *$ & $2.64 \pm 0.52 *$ & $2.67 \pm 0.51 *$ \\
\hline & GroupIII & $2.57 \pm 0.48$ & $3.05 \pm 0.31 *$ & $3.11 \pm 0.33 *$ & $3.13 \pm 0.33 *$ \\
\hline \multirow{3}{*}{ LPO } & GroupI & $2.72 \pm 0.20$ & $2.54 \pm 0.41$ & $2.71 \pm 0.20$ & $2.70 \pm 0.20$ \\
\hline & Group II & $4.15 \pm 0.58$ & $4.13 \pm 0.58$ & $4.08 \pm 0.62 *$ & $3.76 \pm 0.62 *$ \\
\hline & GroupIII & $4.90 \pm 0.73$ & $3.60 \pm 0.73 *$ & $2.90 \pm 0.73 *$ & $2.20 \pm 0.73 *$ \\
\hline \multirow{3}{*}{ FBS } & Group I & $87.70 \pm 5.47$ & $88.13 \pm 5.12$ & $87.83 \pm 5.59$ & $87.17 \pm 5.16$ \\
\hline & Group II & $171.26 \pm 10.59$ & $162.35 \pm 11.39$ & $157.96 \pm 11.89$ & $150.13 \pm 11.98^{* * *}$ \\
\hline & Group III & $180.91 \pm 10.68$ & $158.43 \pm 10.06$ & $155.04 \pm 10.55$ & $146.04 \pm 10.53^{* * *}$ \\
\hline \multirow{3}{*}{ HbA1c } & Group I & $5.28 \pm 0.22$ & $5.32 \pm 0.25$ & $5.31 \pm 0.25$ & $5.30 \pm 0.24$ \\
\hline & Group II & $7.40 \pm 0.43$ & $7.38 \pm 0.43$ & $7.35 \pm 0.44$ & $5.79 \pm 0.41^{*}$ \\
\hline & Group III & $7.60 \pm 0.42$ & $7.33 \pm 0.40$ & $7.30 \pm 0.42$ & $5.26 \pm 0.34^{*}$ \\
\hline
\end{tabular}

Antioxidant enzymes, FBS, HbA1c parameters among groups. Group I(controls)- Normal healthy volunteers , Group II- Type 2 diabetics received Metformin, Group III- Type 2 diabetics received Metformin + Enalapril for a period of 12 weeks. Data are expressed as mean \pm SD. ${ }^{*} \mathrm{p}<0.05,{ }^{* *} \mathrm{p}<0.01, * * * \mathrm{p}<0.001$ when compared to control. FBS- fasting blood sugar, HbA1c - glycated haemoglobin, SOD - superoxide dismutase.

\section{DISCUSSION}

Oxidative stress may play an important role in the onset and progression of diabetes and its complications. This suggests the possibility that such complications can be prevented and treated with antioxidants. The results obtained showed that newly diagnosed type 2 diabetics treated for 12 weeks with Enalapril and Metformin had significantly, reduced free radicals when compared with Metformin group and it goes positively with FBS and HbA1c (Table 1). This indicates that oxidative stress measured as MDA influenced by glycaemic control in type 2 diabetics. In type 2 diabetics, if glycaemic control improves, the oxidative stress indicators such as MDA will partially decrease. There is growing evidence that excess generation of highly reactive free radicals, largely due to hyperglycaemia causes oxidative stress, which further exacerbates the development and progression of type 2diabetes and its complications. ${ }^{17}$ One study in Netherland stated that for patients with type 2 diabetes, treatment with an ACE inhibitor prevented the occurrence or progression of diabetic kidney disease and is highly cost-effective if we start as early after diagnosis. ${ }^{18}$ Antioxidant therapy remains a good therapy for DM2, but it needs to be explored more. AngiotensinII blockage by the angiotensin converting enzyme 
inhibitors(ACEI's) shows to increase the activity of antioxidant enzymes during the diabetes. ${ }^{19}$ ACE inhibitors represent a novel antioxidant strategy that targets vascular NADPH oxidase. ACE inhibition limits the stimulation of vascular $\mathrm{NAD}(\mathrm{P}) \mathrm{H}$ oxidase, thereby preventing the increased superoxide flux associated with activation of the renin-angiotensin system, superoxide reacts with Nitric oxide (NO). The Nitric oxide is known to inhibit the activity of $\mathrm{NAD}(\mathrm{P}) \mathrm{H}$ oxidase. ${ }^{20,21} \mathrm{ACE}$ inhibition should also inhibit lipid peroxidation through reduced formation of peroxynitrite; this notion is consistent with observations that angiotensin II induces lipid peroxidation in experimental animals. Metformin has antioxidant properties which are not fully characterized. It reduces reactive oxygen species (ROS) by stimulating the mitochondrial respiration and decreases advanced glycosylation end product (AGE) indirectly and directly through reduction of hyperglycemia and an insulin-dependent mechanism respectively. ${ }^{22,23}$ There is some evidence that Metformin also has a beneficial effect on some components of the antioxidant defence system. It causes an increase in reduced glutathione and upregulate uncoupled proteins 2 (UCP2) in adipose tissue. ${ }^{24}$ The results of the present study confirmed that Enalapril and Metformin has positive impact on oxidative stress and glycaemic control influences free radical production. It is thus suggested that the inclusion of adjuvant antioxidant micronutrients to hypoglycaemic agents in the management of diabetes, might go a long way in the reduction of associated complications.

\section{CONCLUSION}

Oxidative stress has been demonstrated to have a major impact in the progression of diabetes, including impairment of insulin action, and elevation of the complication incidence. Antioxidants have already shown to be prospective in the treatment of Type 2 diabetes. Treatments for diabetes that are specified in this study to prevent against oxidative stress are due to the use of drugs with antioxidant property. The results of this study showed that there is a positive correlation between oxidative stress and glycemic control and both Enalapril and Metformin combination possess significant effect on combating oxidative stress than Metformin alone. Further large scale randomized trials are required to confirm this study results.

Funding: No funding sources Conflict of interest: None declared

Ethical approval: The study was approved by the Institutional Ethics Committee

\section{REFERENCES}

1. Asif M. The prevention and control the type-2 diabetes by changing lifestyle and dietary pattern. J Educ Health Promot. 2014;3:1.
2. Gerald IS, Morino K, Petersen KF, Sono S, Choi CS, Varman TS et al. Regulation of mitochondrial biogenesis by lipoprotein lipase in muscle of insulinresistant offspring of parents with type 2 diabetes. Diabetes. 2012;61(4):877-87.

3. William IS, Yorek MA. Mitochondrial dysfunction in diabetes: from molecular mechanisms to functional significance and therapeutic opportunities. Antioxid Redox Signal. 2010;12(4):537-57.

4. Lu H, Koshkin V, Allister EM, Gyulkhandanyan $\mathrm{AV}$, Wheeler MB. Molecular and metabolic evidence for mitochondrial defects associated with beta-cell dysfunction in a mouse model of type 2 diabetes. Diabetes. 2010;59(2):448-59.

5. Rury RH, Sanjoy KP, Angelyn Bethel M, Matthews DR, Andrew H, Neil W et al. 10-Year follow-up of intensive glucose control in type 2 diabetes. $\mathrm{N}$ Engl $\mathrm{J}$ Med. 2008;359:1577-89.

6. Alqahtani N, Waseem Abdul GK, Mohamed HA, Yasar Albushra AR. Use of glycated hemoglobin in the diagnosis of diabetes mellitus and pre-diabetes and role of fasting plasma glucose, oral glucose tolerance test. Int J Prev Med. 2013;4(9):1025-102.

7. Azam M, Marwood L, Ismail K, Evans T, Sivaprasad $\mathrm{S}$, Winkley $\mathrm{K}$ et al. Diabetes complications at presentation and one year by glycated haemoglobin at diagnosis in a multiethnic and diverse socioeconomic population: results from the south london diabetes study. J Diabetes Res. 2015;2015:587673.

8. George MM, Copeland KC. Current treatment options for type 2 diabetes mellitus in youth: today's realities and lessons from the TODAY. Curr Diabetes Rep. 2013;13(1):72-80.

9. Aguayo Rojas LB, Gomes MB. Metformin: an old but still the best treatment for type 2 diabetes. Diabetol Metab Syndr. 2013;5:6.

10. Bajaj S, Khan A. Antioxidants and diabetes. Indian J Endocrinol Metab. 2012;16(12):267-71.

11. Xie Q, Hao CM, Ji L, Hu D, Zhu T, Li X et al. ACEI/ARB underused in patients with type 2 diabetes in chinese population (CCMR-3B Study). PLoS One. 2015;10(2):e011697.

12. Steven S, Münzel T, Daiber A. Exploiting the pleiotropic antioxidant effects of established drugs in cardiovascular disease. Int $\mathrm{J}$ Mol Sci. 2015;16(8):18185-223.

13. Tavafi M. Diabetic nephropathy and antioxidants. J Nephropathol. 2013;2(1):20-7.

14. Rao YM, lakshmi IA, Saroja M. Effect of ACE inhibitors on antioxidant status in streptozotocin induced diabetic rats. Asian J Pharmaceu Clin Res. 2011;4(1):134-7.

15. Zhu A, Romero R, Petty HR. An enzymatic colorimetric assay for glucose-6-phosphate. Anal Biochem. 2011;419(2):266-7.

16. Alam N, Rafiquzzaman. Review on in-vivo and invitro methods evaluation of antioxidant activity. Saudi Pharmaceu J. 2013;21(2):143-52. 
17. Giacco F, Brownlee M. Oxidative stress and diabetic complications. Circ Res. 2010;107(9):1058-70.

18. Adarkwah CC, Gandjour A, Akkerman M, Silvia M. Cost-effectiveness of angiotensin-converting enzyme inhibitors for the prevention of diabetic nephropathy in the Netherlands-a markov model. Plos one. 2011;6(10):e26139.

19. Moinuddin G, Mohammed NI, Kulkarni KS, Kulkarni C. Modulation of haemodynamics, endogeneous antioxidant enzymes, and pathophysiological changes by selective inhibition of angiotensin II type 1 receptors in pressure-overload rats. Cardiovasc J Afr. 2013;24(3):58-65.

20. Yacoub R, Campbell KN. Inhibition of RAS in diabetic nephropathy. Int $\mathrm{J}$ Nephrol Renovasc Disease. 2015;8:29-40.

21. Kanwar YS, Sun L, Xie P, Liu F, Chen S. A glimpse of various pathogenetic mechanisms of diabetic nephropathy. Annu Rev Pathol. 2011;6:395-423.
22. Ahmad S, Shahab U, Baig MH, Khan MMS, Khan S, Srivastava AK. Inhibitory effect of metformin and pyridoxamine in the formation of early, intermediate and advanced glycation end-products. PLoS One. 2013;8(9):e72128.

23. Vlassara H, Uribarri J. Advanced glycation end products (age) and diabetes: cause, effect, or both? Curr Diab Rep. 2014;14(1):453.

24. Rupprecht A, Brauer AU, Chenko AS, Goyn J, Hilse $\mathrm{KE}$, Irina $\mathrm{G}$ et al. Quantification of uncoupling protein 2 reveals its main expression in immune cells and selective up-regulation during T-Cell proliferation. Plos one. 2012;7(8):e41406.

Cite this article as: Sathiya Vinotha AT, Ranjani R. A prospective randomized study: role of enalapril on oxidative stress in newly diagnosed type 2 diabetes mellitus and its correlation with glycaemic status. Int J Basic Clin Pharmacol 2017;6:874-8. 